\title{
Association between Carotid Plaque Features on CTA and Cerebrovascular Ischemia: A Systematic Review and Meta-Analysis
}

\author{
(D) H. Baradaran, (D). Al-Dasuqi, (D)A. Knight-Greenfield, (D)A. Giambrone, DD. Delgado, (DE.J. Ebani, (D) H. Kamel, and (D) A. Gupta
}

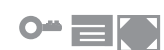

\begin{abstract}
BACKGROUND: CTA is a widely available imaging examination that may allow the evaluation of high-risk carotid plaque features.

PURPOSE: Our aim was to evaluate the association between specific carotid plaque features on CTA and ipsilateral cerebrovascular ischemia.

DATA SOURCES: We performed a systematic review of Ovid MEDLINE, Ovid Embase, Scopus, and the Cochrane Library from inception to March 2016 for articles that evaluated the relationship between CTA-detected carotid plaque features and ischemic events, defined as ipsilateral ischemic stroke or transient ischemic attack.
\end{abstract}

STUDY SELECTION: Sixteen studies were ultimately included after screening 12,557.

DATA ANALYSIS: Two readers recorded data from each study and assessed the study quality with all disagreements resolved by a third reader. A random-effects OR was used to evaluate the association between cerebrovascular ischemia and each of the evaluated plaque features.

DATA SYNTHESIS: We found significant positive relationships with cerebrovascular ischemia for the presence of soft plaque (OR, 2.9; $95 \% \mathrm{Cl}, 1.4-6.0$ ), plaque ulceration (OR, 2.2; 95\% Cl, 1.4-3.4), and increased common carotid artery wall thickness (OR, 6.2; 95\% Cl, 2.5-15.6). We found a significant negative relationship between calcified plaque and ipsilateral ischemia (OR, $0.5 ; 95 \% \mathrm{Cl}, 0.4-0.7)$.

LIMITATIONS: We found heterogeneity in the existing literature secondary to lack of standardized plaque features and clinical definitions.

CONCLUSIONS: Soft plaque, plaque ulceration, and increased common carotid artery wall thickness on CTA are associated with ipsilateral cerebrovascular ischemia, while calcified plaque is negatively associated with downstream ischemic events.

ABBREVIATION: US = ultrasound

$\mathbf{G}^{\mathrm{i}}$ iven recent improvement in medical treatment, patients with asymptomatic carotid artery stenosis receiving modern intensive medical therapy now face an annual risk of stroke of $\sim 1 \%$. ${ }^{1}$ Given the limitations of stenosis measurements alone in identifying patients at highest risk of ischemic stroke, recent in-

Received January 17, 2017; accepted after revision June 30.

From the Department of Radiology (H.B., K.A.-D., A.K.-G., E.J.E., A.G.); Clinical and Translational Neuroscience Unit (H.B., H.K., A.G.), Feil Family Brain and Mind Research Institute (H.K., A.G.); Department of Healthcare Policy and Research (A.G.); Samuel J. Wood Library and C.V. Starr Biomedical Information Center (D.D.); and Department of Neurology (H.K.), Weill Cornell Medicine, New York, New York.

Dr Ajay Gupta is supported by the National Institutes of Health/National Center for Advancing Translational Sciences grant No. KL2TR000458, and Dr Hooman Kamel is supported by the National Institutes of Health/National Institute of Neurological Disorders and Stroke grant No. K23NS082367.

Please address correspondence to Ajay Gupta, MD, 525 East 68th St, Box 141, Starr 8A, New York, NY; e-mail: ajg9004@med.cornell.edu vestigations using vessel wall imaging techniques have attempted to provide more detailed characterization of vulnerable plaque features. Specific plaque features, such as intraplaque hemorrhage on MR imaging and echolucent plaque on ultrasound (US), have been identified as risk factors for future ischemic stroke, which, when considered along with stenosis severity and other factors, may help to identify those patients most likely to benefit from surgical revascularization procedures. ${ }^{2,3}$

CTA is a potentially attractive tool for plaque imaging because it is less operator-dependent than US and is more quickly performed and more widely available than MR imaging. ${ }^{4}$ Easily identifiable plaque

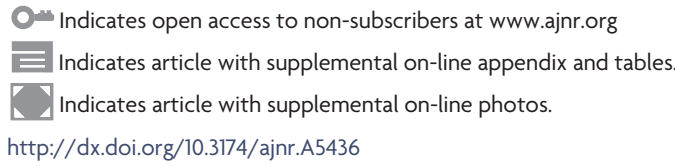

AJNR Am J Neuroradiol 38:2321-26 Dec 2017 www.ajnr.org 
features on CTA such as the presence of low attenuation, calcification, and plaque ulceration have been histopathologically validated as markers of high-risk plaque features. ${ }^{4-6}$ Although CTA has significant potential to evaluate plaque features, small studies have not reached a consensus regarding plaque features, and high-resolution 3T MR imaging techniques have been favored. We aimed to perform a systematic review and meta-analysis to evaluate the association between multiple specific carotid artery plaque features seen on CTA and cerebrovascular ischemic events.

\section{MATERIALS AND METHODS}

We performed this systematic review and meta-analysis according to the guidelines from the Meta-Analysis of Observational Studies in Epidemiology group ${ }^{7}$ and the Preferred Reporting Items for Systematic Reviews and Meta-Analyses (PRISMA) statement. ${ }^{8}$

\section{Data Searches}

A medical librarian performed a comprehensive literature search in multiple electronic databases, including Ovid MEDLINE, Ovid Embase, Scopus, and the Cochrane Library from inception to March 9, 2016. We searched first in Ovid MEDLINE and then adapted subject headings and key words for other databases and identified additional records using the "Cited by" and "View references" features in Scopus (see On-line Appendix for search methodology details).

\section{Study Selection and Eligibility}

We included studies evaluating the association between various plaque features on CTA and symptomatic cerebrovascular ischemic events, defined as either prior or future transient ischemic attack or stroke in the vascular territory supplied by the index carotid artery. Specific inclusion criteria were the following: 1) studies that evaluated patients with plaque in the extracranial internal carotid artery; 2) studies using CTA of the common and cervical internal carotid arteries to assess specific plaque features; 3) studies that correlated the questioned plaque features with symptomatic status, defined as either stroke or transient ischemic attack in the vascular territory supplied by the index carotid artery; 4) studies that included asymptomatic control carotid arteries, either by comparing with the asymptomatic contralateral carotid artery (within-subject controls) or asymptomatic subjects (between-subject controls). If it appeared that authors published data from a single cohort or medical center more than once, the article with the largest sample size was included to minimize duplicate or overlapping samples. We attempted to contact the corresponding authors for additional details when necessary.

\section{Data Extraction}

All potentially eligible titles and abstracts were reviewed by a single reader. Two independent readers screened articles in their entirety to determine eligibility for inclusion. Data were extracted by 2 independent readers using a prespecified data-collection template. A third reader resolved any disagreements about data extraction. The readers extracted the following data: study design; basic study demographics for included patients, including risk factors for stroke; definitions of ischemic stroke; specific CTA imaging techniques; and definitions of plaque features.
We also answered specific questions to evaluate potential selection, detection, reporting, or confounding bias using a risk-ofbias assessment like that in previously published meta-analyses. ${ }^{2,9}$ The risk of bias was assessed by consensus among 3 readers.

\section{Data Analysis}

Meta-analyses of the individual study odds ratios were conducted with StatsDirect statistical software (Version 2.7.9; July 9, 2012; http://www.statsdirect.com). Each pooled OR was calculated with a random-effects (DerSimonian and Laird) model, and forest plots were generated to display the individual study odds ratios and the pooled OR. Random-effects models were used to combine the studies because of the potential of variability in the outcome of interest among the studies. To assess the combinability of the OR, we calculated the $P$ value from the Cochrane $Q$ and $I^{2}$ statistical heterogeneity tests. The results of each study were expressed as an OR with a 95\% confidence interval. For each metaanalysis with $>3$ studies, the presence of publication bias was evaluated through a funnel plot. The Begg and Mazumdar rankcorrelation test was used to statistically assess the presence of publication bias. All $P$ values $<.05$ were considered significant.

\section{RESULTS}

\section{Study Characteristics}

After screening 12,557 titles and abstracts, we identified 20 studies that were ultimately included in the systematic review (On-line Fig 1). ${ }^{6,10-28}$ Of the 20 articles meeting the inclusion criteria for systematic review, 13 were retrospective, cross-sectional studies $^{6,10-12,14-18,20,21,24,28}$ and 7 were prospective, cross-sectional studies. ${ }^{13,19,22,23,25-27}$ The time interval between the onset of ischemic symptoms and CTA ranged from 2 weeks to 6 months for those studies that provided these data (On-line Table 1). ${ }^{14,20,22,23}$ We found no studies evaluating the association between CTA plaque features and future ischemic events. Seven studies were performed in the United States ${ }^{6,10,11,18,20,24,28}$; 3, in Japan ${ }^{14,22,23}$; 3 , in Italy ${ }^{15-17} ; 2$, in the Netherlands ${ }^{19,27}$; and 1 each, in Canada, ${ }^{21}$ France, ${ }^{13}$ China, ${ }^{26}$ Germany, ${ }^{25}$ and Spain. ${ }^{12}$ The mean age of patients in the included studies ranged from 62 to 75.1 years. All studies had a preponderance of male subjects with a percentage range of men from $53.7 \%$ to $92.3 \%$. There was a range of degree of ICA stenosis, with 6 studies requiring patients to have at least $50 \%$ stenosis, ${ }^{11,13,14,16,23,25} 2$ studies requiring patients to have at least $60 \%$ stenosis, ${ }^{10,12}$ and 2 studies requiring patients to have at least $70 \%$ stenosis. ${ }^{20,29}$ Six studies evaluated all patients with CTA examinations regardless of their degree of stenosis, ${ }^{15,18,19,21,24,26}$ while 2 studies focused only on patients with mild-to-moderate $(30 \%-69 \%)$ stenosis, ${ }^{22,27}$ and 1 study focused only on patients with moderate $(50 \%-69 \%)$ stenosis. ${ }^{28}$ An additional study included those patients with at least $70 \%$ stenosis, symptomatic patients with at least $50 \%$ stenosis, and all symptomatic patients with evidence of plaque ulceration regardless of the degree of stenosis (On-line Table 2). ${ }^{11}$

Twelve studies were performed on at least a 16-detector row helical CT scanner, ${ }^{11,14,18-20,22-24,26-29}$ while only 7 studies included patients who may have been scanned on a 4 - or 8 -detector row CT scanner. ${ }^{10,12,13,15-17,21}$ One study did not provide the relevant number of rows (On-line Table 3$).^{25}$ 
Of those 20 studies included for systematic review, 16 studies were eligible for meta-analysis in which 2624 patients with 3933 unique carotid arteries were analyzed. The 4 studies excluded from the meta-analysis were not amenable to calculating pooled standardized mean differences because of methodologic differences in calculating volumes, variability in plaque-feature definitions, and small sample sizes for each calculation and included studies that quantitatively evaluated the volume of soft/noncalcified plaque, the volume of calcified plaque, and Hounsfield units (On-line Table 4).

Although specific definitions varied, patients were symptomatic if they had a prior ischemic stroke or TIA in the vascular territory supplied by the carotid artery in question (On-line Table 1). Strokes were generally defined as the patient having had a persistent episode of neurologic dysfunction with confirmatory imaging in the distribution of the carotid artery, while TIAs were defined as brief episodes ( $<24$ hours) of neurologic dysfunction, also within the carotid artery distribution.

\section{Definitions of Plaque Features}

From the included studies, we were able to collect the actual number of cerebrovascular events for patients with each plaque feature to calculate pooled odds ratios expressing the strength of association between recent ischemic events and the following 4 plaque features: 1) the presence of low attenuation or "soft" plaque, 2) the presence of calcified plaque, 3) plaque ulceration, and 4) common carotid artery wall thickness (On-line Table 5). Soft plaques were defined as having low density or lipid-rich cores, with 4 studies using a specific threshold of Hounsfield units of $<50$ or $60 .^{10,17,25,26}$ The presence of calcified plaque was defined as extensively calcified plaque with Hounsfield units of $>120$ or 130. ${ }^{10,17,25,26}$ Specific definitions for plaque ulceration varied among studies, but it was generally defined as extension of contrast material beyond the vascular lumen into the plaque. Last, common carotid artery wall thickness was defined as thickening of the common carotid artery wall and was dichotomized by the authors of the included studies using various thresholds.

\section{Meta-Analysis Results}

We performed 4 separate meta-analyses. For the meta-analyses evaluating the association between low-attenuation plaque, plaque ulceration, and increased common carotid artery wall thickness, we included 1801, 2883, and 307 arteries in each metaanalysis, respectively (On-line Table 6). We found a significant positive association between soft or low-attenuation plaque, plaque ulceration, and increased common carotid artery wall thickness and the presence of recent ipsilateral stroke or TIA, with pooled ORs of 2.92 (95\% CI, 1.41-6.04; $P=.004), 2.20$ (95\% CI, 1.43-3.40; $P<.001$ ), and 6.19 (95\% CI, 2.47-15.55; $P<.001$ ), respectively (Fig 1). We also analyzed 2004 arteries to determine the association of the presence of a calcified plaque and downstream cerebrovascular ischemic symptoms and found a negative association with a pooled OR of 0.536 (95\% CI, 0.384-0.749; $P<$.001) (Fig 1). Measures of study heterogeneity and publication bias for the included meta-analyses (Table) demonstrated moderate heterogeneity. Publication bias (On-line Fig 2) was only statistically significant for plaque ulceration studies.

\section{Assessment of the Quality and Bias of the Included Studies}

Our quality and bias assessment questionnaire (On-line Table 7) demonstrated that the inclusion and exclusion criteria were adequately described in all the included studies. All except 1 study included in the meta-analysis had investigators blinded to the symptomatic status of the artery in question, with that single study failing to describe the blinded status of the investigators. ${ }^{19}$ Thirteen of the included studies had $>1$ investigator evaluating the questioned plaque feature, while 3 studies were aided by computer algorithms to assess plaque features. ${ }^{18,22,24}$ Half of the studies reported measures of interreader reproducibility with $\kappa$ values ranging from 0.46 to 1 , depending on the specific plaque feature. ${ }^{11,13,15-17,19,20,27-29}$ Twelve studies evaluated the degree of stenosis in addition to evaluating specific plaque features. ${ }^{6,11,13,14,17-22,24,28}$

\section{DISCUSSION}

In this systematic review and meta-analysis, we found that patients with carotid artery atherosclerotic disease demonstrating CTA evidence of low-attenuation plaque, increased common carotid artery wall thickness, or plaque ulceration are highly associated with the presence of recent ipsilateral ischemic events, while those patients with calcified plaques are associated with fewer ipsilateral ischemic events. Our findings are compatible with studies performed on histopathologic carotid endarterectomy specimens. Low-attenuation or soft plaque on CTA is thought to correspond to the histologically described lipid-rich necrotic core and intraplaque hemorrhage ${ }^{4}$ and has been shown to be associated with increased risk of future stroke on both MR imaging and US. ${ }^{2,3}$ Additionally, plaque ulceration on histopathologic samples and on high-resolution MR imaging has also been associated with symptomatic plaque. ${ }^{2,30,31}$ Increased common carotid artery wall thickness, traditionally measured as intima-media thickness on US, is thought to reflect arterial inflammation and is a predictor of cerebrovascular events in prospective studies. ${ }^{32}$ Conversely, histopathologic studies evaluating echogenic calcified plaque on US have found that densely calcified plaques are less frequently associated with ischemic events and may be a protective plaque feature, perhaps by preventing thrombus aggregation or by affording additional mechanical stability to a plaque surface. ${ }^{3,33}$ Additionally, a previously published systematic review has shown that symptomatic plaques have less calcification than asymptomatic plaques. $^{34}$

Using CTA to evaluate high-risk carotid plaque features has several strengths: First, CTA can be rapidly performed and is commonly available. Unlike high-resolution plaque imaging with MR imaging, CTA plaque imaging does not require lengthy sequences or dedicated equipment such as carotid coils to evaluate plaque features. Additionally, evaluating the presence of soft or calcified plaque, plaque ulceration, or increased common carotid artery wall thickness can be easily performed with high reproducibility without requiring lengthy interpretive time or postprocessing software.

Our study illustrates some methodologic limitations of the existing literature on CTA evaluation of carotid plaque. Our analysis revealed moderate levels of heterogeneity in the meta-analy- 

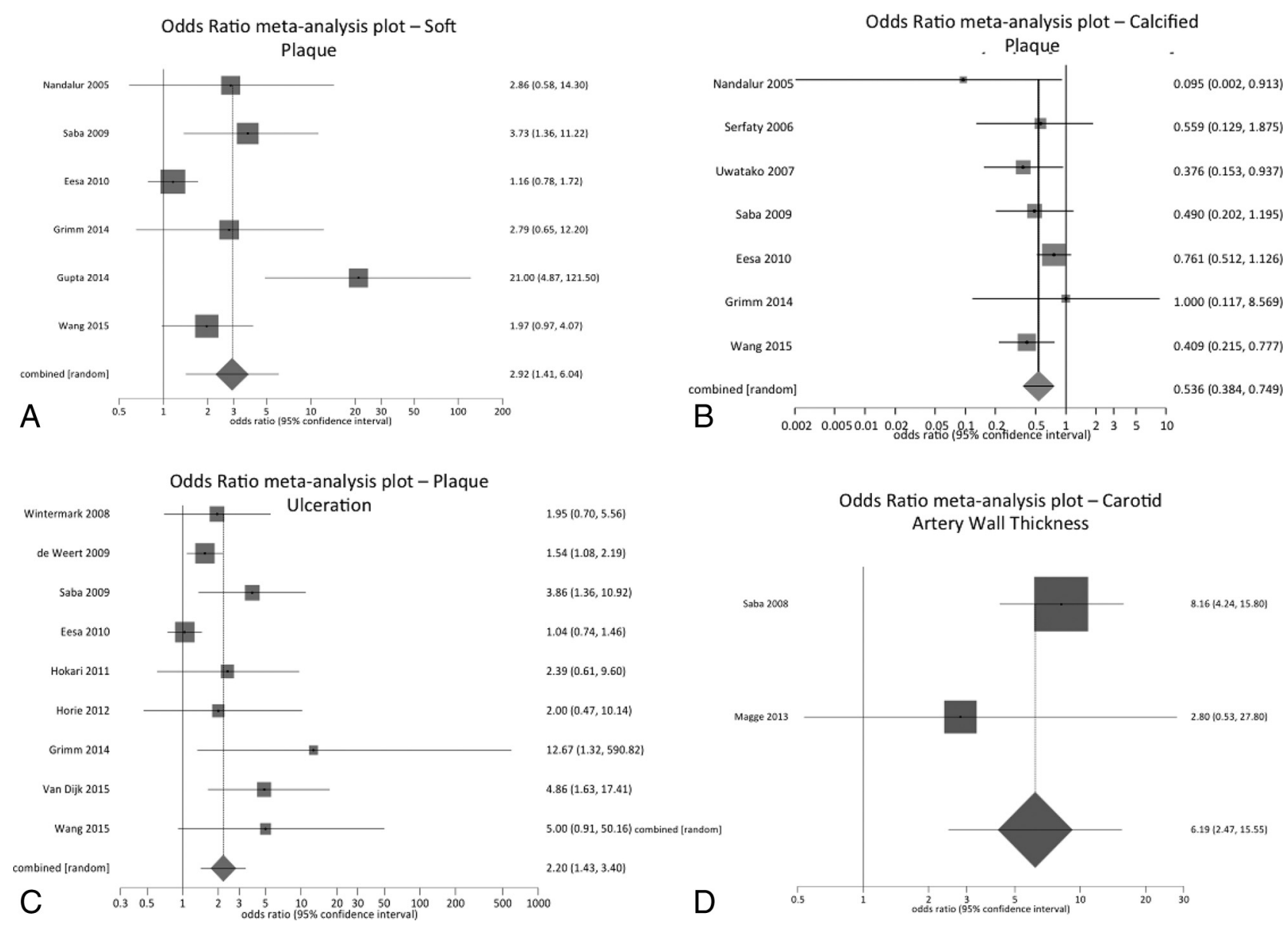

FIG 1. Four separate forest plots of the association between CT angiography-determined plaque characteristics and recent prior ipsilateral ischemic events. Each meta-analysis was calculated with a random-effects model with pooled ORs shown for each forest plot. Each square represents the point estimate of the effect size of the study with the square size being proportional to the inverse of the variance of the estimate and the horizontal lines representing each the $95 \% \mathrm{Cl}$ of each study. The diamond represents the pooled estimate with the width of the diamond representing the pooled $95 \% \mathrm{Cl}$.

\section{Heterogeneity and publication bias measures}

\begin{tabular}{|c|c|c|c|c|c|}
\hline & \multicolumn{3}{|c|}{ Measures of Heterogeneity } & \multicolumn{2}{|c|}{ Publication Bias } \\
\hline & $\mathrm{I}^{2}$ & Cochran Q & $P$ Value & Kendall $T$ & $P$ Value \\
\hline Soft-plaque studies & $76.3 \%$ & 21.13 & .001 & 0.47 & .27 \\
\hline Calcified-plaque studies & $19.8 \%$ & 7.49 & .28 & 0.14 & .56 \\
\hline Plaque ulceration studies & $61.1 \%$ & 20.56 & .008 & 0.389 & .018 \\
\hline Carotid artery wall thickness studies & $34.6 \%$ & 1.52 & .22 & NA & NA \\
\hline
\end{tabular}

Note:-NA indicates not applicable.

ses evaluating soft plaque and plaque ulceration, which may have been secondary to between-study variability in the definitions of CTA plaque features and differences in how studies adjudicated and defined stroke or TIA. Using a random-effects rather than a fixed-effects model, we could statistically account for this heterogeneity and still show strong associations between each plaque feature and cerebrovascular ischemia. We believe that increased standardization of plaque feature definitions and more consistently applied, uniform definitions of ischemic events are warranted for future studies. Additionally, risk of bias for each article was determined subjectively by consensus among 3 readers. The subjectivity involved in assessing the risk of bias is inherent to systematic reviews and meta-analyses. We also detected the possibility of publication bias in our meta-analysis of plaque ulceration, which raises the possibility that negative studies were not published. However, the evaluation of publication bias is limited, given the small number of studies published on the CTA characterization of plaque ulceration, and future studies are warranted to examine whether such a bias exists.

Our systematic review and meta-analyses also revealed knowledge gaps that future studies should seek to clarify. First, we found that investigators have, to date, evaluated only the relationship between specific plaque features and recent prior ischemic symptoms, rather than future stroke. Although longitudinal MR imaging studies have shown that high-risk carotid plaque features such as intraplaque hemorrhage do not change significantly during a 1 -year period in symptomatic patients, ${ }^{35}$ prospective studies evaluating future stroke risk are needed if CTA plaque analysis is to play a greater role in primary stroke prevention. It is possible that a more directly applicable use of the results of our study may allow 
clinicians to improve their confidence in identifying a culprit lesion after a stroke of uncertain etiology has occurred so that optimal secondary stroke prevention measures can be initiated. ${ }^{36,37}$ Second, we found that the time interval between the onset of ischemic symptoms and CTA for the evaluation of plaque features was relatively inconsistent among included studies. Third, many studies did not include precise descriptions of how causation of ischemic stroke was attributed to a given ICA. Prospective studies with standardized protocols and definitions evaluating the predictive value of these plaque features for ischemic symptoms are warranted to improve the clinical usefulness of carotid plaque CTA.

\section{CONCLUSIONS}

Our systematic review and meta-analyses suggest that plaque features such as the presence of soft plaque, plaque ulceration, or increased common carotid artery wall thickness seen on CTA are strongly positively associated with cerebrovascular ischemic events and that the presence of calcified plaque is negatively associated with prior ischemic events. Routine assessment of these plaque features on CTA may be complementary to measuring degree of luminal stenosis and aid in identifying high-risk plaque features.

Disclosures: Ajay Gupta—RELATED: Grant: National Institutes of Health*. *Money paid to the institution.

\section{REFERENCES}

1. Raman G, Moorthy D, Hadar N, et al. Management strategies for asymptomatic carotid stenosis: a systematic review and meta-analysis. Ann Intern Med 2013;158:676-85 CrossRef Medline

2. Gupta A, Baradaran H, Schweitzer AD, et al. Carotid plaque MRI and stroke risk: a systematic review and meta-analysis. Stroke 2013;44: 3071-77 CrossRef Medline

3. Gupta A, Kesavabhotla K, Baradaran H, et al. Plaque echolucency and stroke risk in asymptomatic carotid stenosis a systematic review and meta-analysis. Stroke 2015;46:91-97 CrossRef Medline

4. Wintermark M, Jawadi SS, Rapp JH, et al. High-resolution CT imaging of carotid artery atherosclerotic plaques. AJNR Am J Neuroradiol 2008;29:875-82 CrossRef Medline

5. Trelles M, Eberhardt K, Buchholz M, et al. CTA for screening of complicated atherosclerotic carotid plaque: American Heart Association type VI lesions as defined by MRI. AJNR Am J Neuroradiol 2013;34:2331-37 CrossRef Medline

6. Gupta A, Baradaran H, Mtui EE, et al. Detection of symptomatic carotid plaque using source data from MR and CT angiography: a correlative study. Cerebrovasc Dis 2015;39:151-61 CrossRef Medline

7. Stroup DF, Berlin JA, Morton SC, et al. Meta-analysis of observational studies in epidemiology: a proposal for reporting; MetaAnalysis Of Observational Studies in Epidemiology (MOOSE) group. JAMA 2000;283:2008-12 CrossRef Medline

8. Liberati A, Altman DG, Tetzlaff J, et al. The PRISMA statement for reporting systematic reviews and meta-analyses of studies that evaluate health care interventions: explanation and elaboration. PLoS Med 2009;6:e1000100 CrossRef Medline

9. Gupta A, Giambrone AE, Gialdini G, et al. Silent brain infarction and risk of future stroke: a systematic review and meta-analysis. Stroke 2016;47:719-25 CrossRef Medline

10. Nandalur KR, Baskurt E, Hagspiel KD, et al. Calcified carotid atherosclerotic plaque is associated less with ischemic symptoms than is noncalcified plaque on MDCT. AJR Am J Roentgenol 2005;184: 295-98 CrossRef Medline

11. Nandalur KR, Hardie AD, Raghavan P, et al. Composition of the stable carotid plaque: insights from a multidetector computed tomography study of plaque volume. Stroke 2007;38:935-40 CrossRef Medline

12. Miralles M, Merino J, Busto M, et al. Quantification and characterization of carotid calcium with multi-detector CT-angiography. Eur J Vasc Endovasc Surg 2006;32:561-67 CrossRef Medline

13. Serfaty JM, Nonent M, Nighoghossian N, et al; CARMEDAS Study Group. Plaque density on CT, a potential marker of ischemic stroke. Neurology 2006;66:118-20 CrossRef Medline

14. Uwatoko $\mathrm{T}$, Toyoda $\mathrm{K}$, Inoue $\mathrm{T}$, et al. Carotid artery calcification on multislice detector-row computed tomography. Cerebrovasc Dis 2007;24:20-26 CrossRef Medline

15. Saba L, Sanfilippo R, Pascalis L, et al. Carotid artery wall thickness and ischemic symptoms: evaluation using multi-detector-row CT angiography. Eur Radiol 2008;18:1962-71 CrossRef Medline

16. Saba L, Mallarini G. Fissured fibrous cap of vulnerable carotid plaques and symptomaticity: are they correlated? Preliminary results by using multi-detector-row CT angiography. Cerebrovasc Dis 2009;27:322-27 CrossRef Medline

17. Saba L, Montisci R, Sanfilippo R, et al. Multidetector row CT of the brain and carotid artery: a correlative analysis. Clin Radiol 2009;64: 767-78 CrossRef Medline

18. Wintermark M, Arora S, Tong E, et al. Carotid plaque computed tomography imaging in stroke and nonstroke patients. Ann Neurol 2008;64:149-57 CrossRef Medline

19. de Weert TT, Cretier S, Groen HC, et al. Atherosclerotic plaque surface morphology in the carotid bifurcation assessed with multidetector computed tomography angiography. Stroke 2009;40:1334-40 CrossRef Medline

20. Romero JM, Babiarz LS, Forero NP, et al. Arterial wall enhancement overlying carotid plaque on CT angiography correlates with symptoms in patients with high grade stenosis. Stroke 2009;40:1894-96 CrossRef Medline

21. Eesa M, Hill MD, Al-Khathaami A, et al. Role of CT angiographic plaque morphologic characteristics in addition to stenosis in predicting the symptomatic side in carotid artery disease. AJNR Am J Neuroradiol 2010;31:1254-60 CrossRef Medline

22. Hokari M, Kuroda S, Yasuda H, et al. Lumen morphology in mild-tomoderate internal carotid artery stenosis correlates with neurological symptoms. J Neuroimaging 2011;21:348-54 CrossRef Medline

23. Horie N, Morikawa M, Ishizaka S, et al. Assessment of carotid plaque stability based on the dynamic enhancement pattern in plaque components with multidetector CT angiography. Stroke 2012;43: 393-98 CrossRef Medline

24. Magge R, Lau BC, Soares BP, et al. Clinical risk factors and CT imaging features of carotid atherosclerotic plaques as predictors of new incident carotid ischemic stroke: a retrospective cohort study. AJNR Am J Neuroradiol 2013;34:402-09 CrossRef Medline

25. Grimm JM, Schindler A, Schwarz F, et al. Computed tomography angiography vs $3 \mathrm{~T}$ black-blood cardiovascular magnetic resonance for identification of symptomatic carotid plaques. J Cardiovasc Magn Reson 2014;16:84 CrossRef Medline

26. Wang P, Wang Y, Zhang G, et al. Study on the carotid atherosclerotic plaque of patients suffering from ischemic cerebrovascular disease by 64 slices CT. Eur Rev Med Pharmacol Sci 2015;19:3480-85 Medline

27. van Dijk A, Truijman M, Hussain B, et al. Intraplaque hemorrhage and the plaque surface in carotid atherosclerosis: the Plaque at RISK study (PARISK). AJNR Am J Neuroradiol 2015;36:2127-33 CrossRef Medline

28. Gupta A, Mtui E, Baradaran H, et al. CT angiographic features of symptom-producing plaque in moderate-grade carotid artery stenosis. AJNR Am J Neuroradiol 2015;36:349-54 CrossRef Medline

29. Gupta A, Baradaran H, Kamel H, et al. Evaluation of computed tomography angiography plaque thickness measurements in highgrade carotid artery stenosis. Stroke 2014;45:740-45 CrossRef Medline 
30. Golledge J, Greenhalgh RM, Davies AH. The symptomatic carotid plaque. Stroke 2000;31:774-81 CrossRef Medline

31. Fisher M, Paganini-Hill A, Martin A, et al. Carotid plaque pathology: thrombosis, ulceration, and stroke pathogenesis. Stroke 2005;36: 253-57 CrossRef Medline

32. Simon A, Gariepy J, Chironi G, et al. Intima-media thickness: a new tool for diagnosis and treatment of cardiovascular risk. J Hypertens 2002;20:159-69 CrossRef Medline

33. Shaalan WE, Cheng H, Gewertz B, et al. Degree of carotid plaque calcification in relation to symptomatic outcome and plaque inflammation. J Vasc Surg 2004;40:262-69 CrossRef Medline

34. Kwee RM. Systematic review on the association between calcifica- tion in carotid plaques and clinical ischemic symptoms. J Vasc Surg 2010;51:1015-25 CrossRef Medline

35. Kwee RM, Truijman MT, van Oostenbrugge RJ, et al. Longitudinal MRI study on the natural history of carotid artery plaques in symptomatic patients. PLoS One 2012;7:e42472 CrossRef Medline

36. Gupta A, Gialdini G, Lerario MP, et al. Magnetic resonance angiography detection of abnormal carotid artery plaque in patients with cryptogenic stroke. J Am Heart Assoc 2015;4:e02012 CrossRef Medline

37. Freilinger TM, Schindler A, Schmidt C, et al. Prevalence of nonstenosing, complicated atherosclerotic plaques in cryptogenic stroke. JACC Cardiovasc Imaging 2012;5:397-405 CrossRef Medline 